\title{
Machine Learning Techniques for Anomaly Detection: An Overview
}

\author{
Salima Omar \\ Universiti Teknologi Malaysia \\ Faculty of Computing
}

\author{
Asri Ngadi \\ Universiti Teknologi Malaysia \\ Faculty of Computing
}

\author{
Hamid H. Jebur \\ Universiti Teknologi Malaysia \\ Faculty of Computing
}

\begin{abstract}
Intrusion detection has gain a broad attention and become a fertile field for several researches, and still being the subject of widespread interest by researchers. The intrusion detection community still confronts difficult problems even after many years of research. Reducing the large number of false alerts during the process of detecting unknown attack patterns remains unresolved problem. However, several research results recently have shown that there are potential solutions to this problem. Anomaly detection is a key issue of intrusion detection in which perturbations of normal behavior indicates a presence of intended or unintended induced attacks, faults, defects and others. This paper presents an overview of research directions for applying supervised and unsupervised methods for managing the problem of anomaly detection. The references cited will cover the major theoretical issues, guiding the researcher in interesting research directions.
\end{abstract}

\section{Keywords}

Supervised Machine Learning, Unsupervised Machine Learning, Network Intrusion Detection.

\section{INTRODUCTION}

Intrusion detection has been studied for approximately 20 years. Intrusions are the activities that violate the information system security policy, and intrusion detection is the identifying intrusions process. Intrusion detection is based on the assumption that the intruder behavior will be significantly diverse from the legitimate behaviors, which facilitates and enables the detection of a lot of non-authorized activities.

Intrusion detection systems are usually used together with other protection systems such as access control and authentication as a second defense line to protect information systems. There are many reasons that make intrusion detection the important parts in the whole defense system. First, many of the traditional systems and applications have been built and developed without taking security seriously into account. Second, computer systems and applications may have flaws or bugs in their design that could be used by intruders to attack the systems or applications. Therefore, the preventive technique may not be as effective as anticipated.

Despite their importance, IDSs are not replacement for preventive security mechanisms, but they complement the other protective mechanisms to enhance the security of the system. Actually, IDSs alone cannot offer sufficient protection for information systems. Therefore, IDSs should be used with other preventive security mechanisms as a part of a total protective system [59]. Intrusion detection systems are classified as a signature detection system and an anomaly detection system. A signature detection system identifies traffic or application data patterns assumed to be malicious, while anomaly detection systems compare activities with "normal baseline. Both signature detection and anomaly detection systems have advantages and drawbacks. The primary advantage of signature detection is that it can detect known attacks fairly for all of the potential attacks against a network. Anomaly detection systems have two main advantages over signature based intrusion detection systems. The first advantage is their capability to detect unknown attacks because they can model the normal operation of a system and detect deviations from this model. The second advantage is the customization ability of the normal activity profiles for every system, application and network. This will increase the difficulty for an attacker to know what activities can be done without getting detected. However, the anomaly detection approach has its drawbacks such as the system complexity, high false alarms and the difficulty of detecting which event triggers those alarms. These are some of many technical challenges that have to be handled before the adoption of anomaly detection systems.

This paper presents an overview of research directions for applying supervised and unsupervised methods for managing the problem of anomaly detection. The rest of this paper is organized as follows. In Section 2, the general architecture of anomaly intrusion detection systems and detailed discussions on the supervised and unsupervised techniques used in anomaly detection are described. Finally, the conclusion of this paper is presented in section 3 .

\section{ANOMALY DETECTION TECHNIQUES}

The general architecture of all anomaly based network intrusion detection systems (A-NIDS) methods is similar. According to [12] and [13], generally, all of them consist of the following basic modules or stages (Fig. 1). These stages are parameterization, training and detection. Parameterization includes collecting raw data from a monitored environment. The raw data should be representative of the system to be modeled, (e.g. Packet data from a network). The training stage seeks to model the system using manual or automatic methods. For the client-server architecture, the server is a host that keeps waiting for incoming connections. When a connection is established between client and server, the server would instantiate a socket, which will be used to instantiate a handler object that runs on a separate thread. These handlers will be kept in a collection object.

The behaviors represented in the model will differ based on the technique used. Detection compares the system generated in the training stage with the selected parameterized data portion. Threshold criteria will be selected to determine anomalous data instance [13]. 


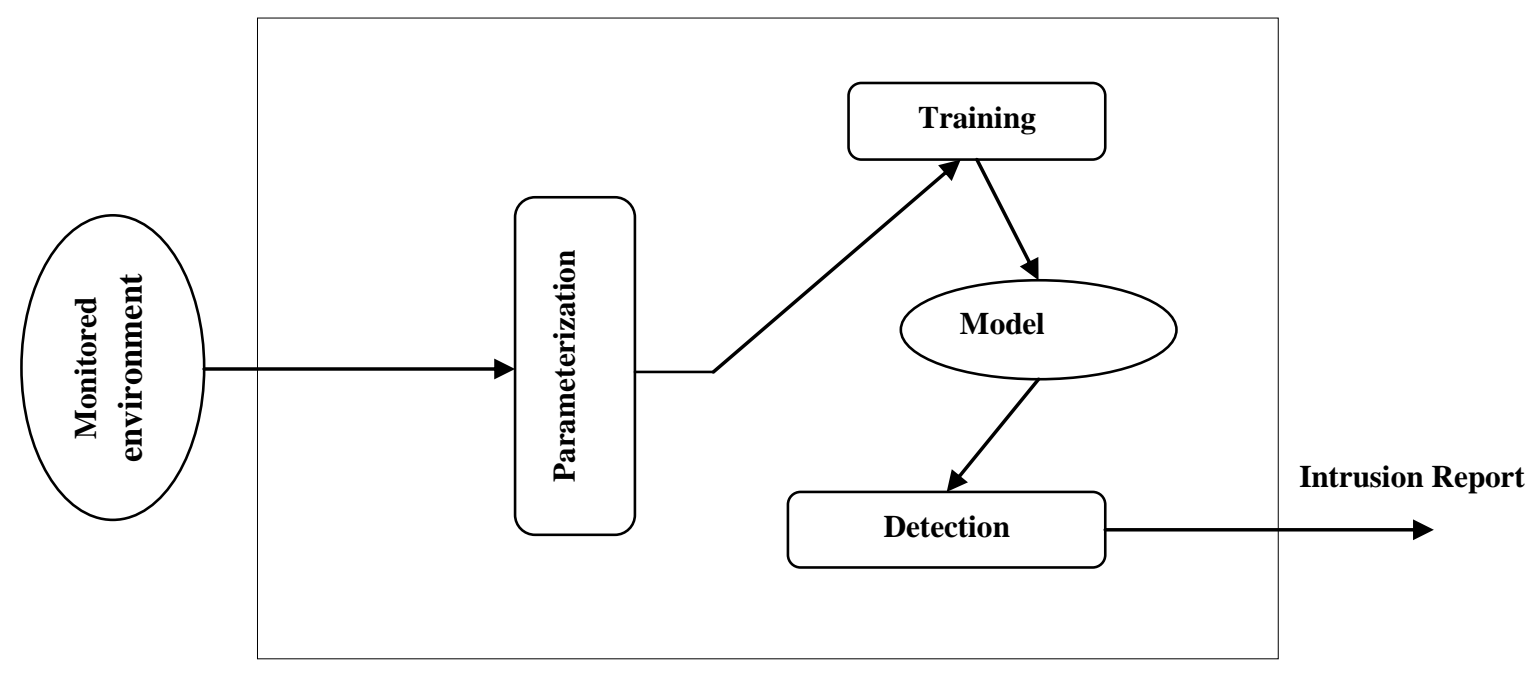

Fig .1 Generic A-NIDS Functional Architecture

Machine learning can build the required model automatically based on some given training data. A motivation for this approach is the availability of the necessary training data, or it can be at least obtained more easily compared to the effort needed to define the model manually. With the increase the complexity and the number of different attacks, machine learning techniques that allow constructing and maintaining anomaly detection system (ADS) with less human intervention look is the only practical approach to achieve the next generation of intrusion detection systems.

Applying machine learning techniques for intrusion detection can automatically build the model based on the training data set, which contains data instances that can be described using a set of attributes (features) and associated labels. The attributes can be of different types such as categorical or continuous.

The attributes nature determines the applicability of anomaly detection techniques. The labels associated with data instances are usually in form of binary values, i.e. normal and anomalous. On the other hand, some researchers have employed various attacks types such as DoS, U2R, R2L and Probe rather than the anomalous label. This learning technique is capable to provide more information about the anomalies types. Anomaly detection techniques include supervised techniques and unsupervised techniques (Fig.2) $[20,55]$.

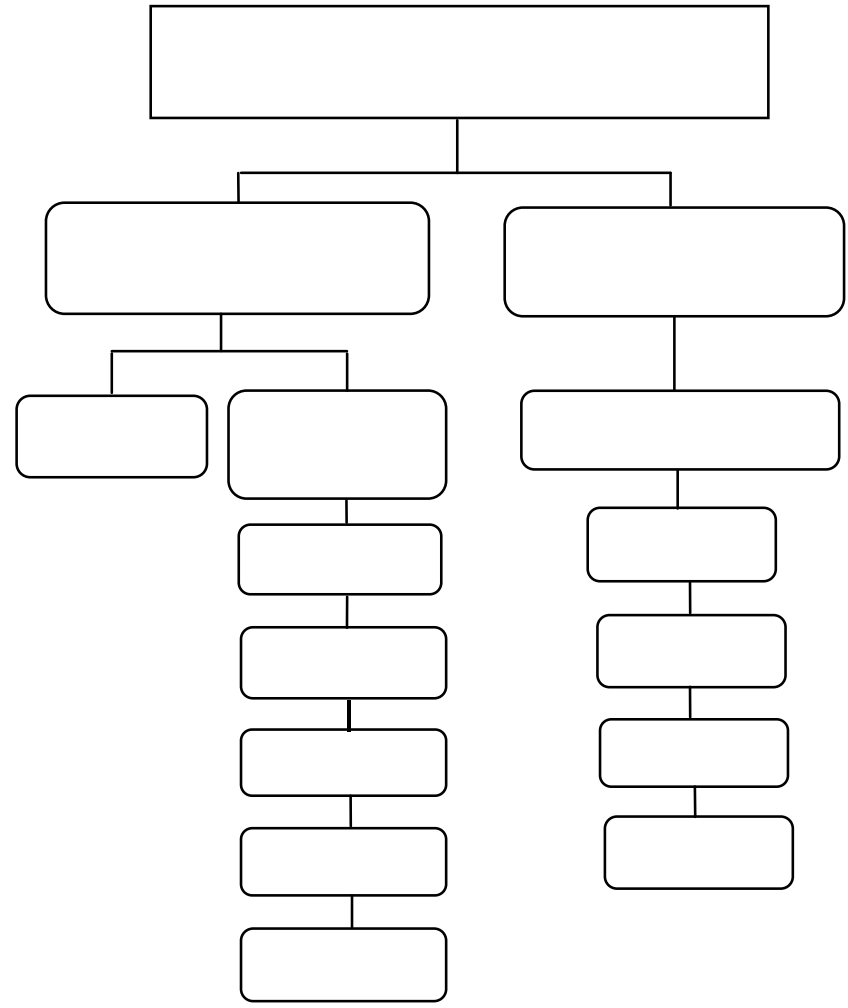

Fig 2: Anomaly Detection Techniques

\subsection{Supervised Anomaly Detection}

Supervised methods (also known as classification methods) required a labeled training set containing both normal and anomalous samples to construct the predictive model. Theoretically, supervised methods provide better detection rate than semi-supervised and unsupervised methods, since they have access to more information. However, there exist some technical issues, which make these methods seem not accurate as they are supposed to be. The first issue is the shortage of a training data set that covers all areas. Moreover, obtaining accurate labels is a challenge and the training sets usually contain some noises that result in higher false alarm rates. The most common supervised algorithms are, Supervised Neural Networks, Support Vector Machines 
(SVM), k-Nearest Neighbors, Bayesian Networks and Decision Tree [60].

\subsection{1 .K -Nearest Neighbor ( $k$-NN)}

$\mathrm{K}$-nearest neighbor (k-NN) is one of the modest and conventional nonparametric techniques for classifying samples [4], [32]. It calculates the approximate distances between various points on the input vectors, and then assigns the unlabeled point to the class of its K-nearest neighbors. In the process of creating $\mathrm{k}-\mathrm{NN}$ classifier, (k) is an important parameter and various $(\mathrm{k})$ values can cause various performances. If $\mathrm{k}$ is very huge, the neighbors, which used for prediction, will consume large classification time and affect the prediction accuracy.

Shailendra and Sanjay [51] introduced a hybrid approach for feature selection, which includes two phases filter and wrapper. The filter phase selects the features with highest information gain and feeds them to the wrapper phase that outputs the final feature subset. The final feature subsets are input to the $\mathrm{K}$-nearest neighbor classifier to classify attacks. This algorithm effectiveness is demonstrated on DARPA KDDCUP99 cyber-attack dataset. Ming. Y [33] suggested a genetic algorithm combined with KNN (k-nearest-neighbor) for feature selection and weighting. All initial 35 features in the training phase were weighted, and the ones of highest weights were selected for testing. Many DoS attacks were applied to evaluate the systems.

\subsubsection{Bayesian Network (BN)}

Heckerman [17] defined a Bayesian as "A Bayesian Network (BN) is a model that encodes probabilistic relationships among variables interest. This technique is generally used for intrusion detection in combination with statistical schemes. It has several advantages, including the capability of encoding interdependencies between variables and of predicting events, as well as the ability to incorporate both prior knowledge and data."

Johansen and Lee [22] stated that a BN system provides a proper mathematical foundation to make straightforward apparently a difficult problem. They have proposed that $\mathrm{BN}$ based IDS should distinguish attacks from normal network activity by comparing metrics of each network traffic sample. Moore and Zuev [35] used a supervised Naive Bayes classifier and 248 flow features to differentiate between different types of application such as packet length and inter arrival times, in addition to numerous TCP header derived features. Correlation-based feature selection was used to define stronger features, and it indicated that only a small subset of fewer than 20 features is needed for accurate classification.

\subsubsection{Supervised Neural Network (NN)}

The NNs learning predict different users and daemons behavior in systems. If they properly designed and implemented, NNs have the capability to address many problems encountered by rule-based approaches. The main NNs advantage is their tolerance to imprecise data and uncertain information, and their ability to conclude solutions from data without having previous knowledge of the regularities in the data. This, in combination with their ability to generalize from learning data, has made them a proper approach to ID. In order to apply this approach to ID, data representing attacks and non-attacks have to be introduced to the NN to adjust automatically network coefficients during the training phase [27]. Multilayer perceptron (MLP) and
Radial basis function (RBF) are the most commonly supervised neural networks used.

Multi Layered Perceptron (MLP). MLP can only classify linearly separable instances sets. If a straight line or plane can be drawn to separate the input instances into their correct categories, input instances are linearly separable and the perceptron will find the solution. If the instances are not linearly separable learning will never reach a point where all instances are classified properly. Multilayered perceptron (Artificial Neural Networks) have been created to try solving this problem [47].

There were researches implement an IDS using MLP, which has the capability of detecting normal and attacks connection as in [54] and [48]. They were implemented using MLP of three and four layers neural network. Moradi and Zulkernine [36], Mohammed et al. [34] used three layers MLP (two hidden layers) not only for detecting normal and attacks connection but also for identifying attack type. Yao et al. [57] proposed Hybrid MLP/CNN neural network, which is constructed in order to enhance the detection rate of timedelayed attacks. While obtaining a similarly detection rate of real-time attacks as the MLP does, the proposed approach can detect time-delayed attacks efficiently with chaotic neuron.

Radial Basis Function Neural Networks (RBF) is another common type of feed forward neural networks. Since they perform classification by measuring distances between inputs and the centers of the RBF hidden neurons, RBF networks are much faster than the time consuming back propagation, and most suitable for problems with large sample size [6]. Research, such as Hofmann et al. [18], Liu et al. [31], Rapaka [45] employed RBFs to learn multiple local clusters for wellknown attacks and for normal events. Other than being a classifier, the RBF network is also used to fuse results from multiple classifiers [6]. It outperformed five different decision fusion functions, such as a Dempster-Shafer combination and weighted majority vote. Jiang et al. [21] introduced a new approach, which combines both misuse and anomaly detections in a hierarchical RBF network. In the first layer, an $\mathrm{RBF}$ anomaly detector defines the event nature if it is normal or anomaly. Anomaly events then pass through a RBF misuse detector chain, where each detector detects a specific type of attack. Un classified anomaly events by any misuse detectors were saved into a database. If enough anomaly events were collected, they were clustered by a C-means clustering algorithm into different groups, which used to train a misuse RBF detector, and added to the misuse detector chain. This mannar leads to detect and lable and all intrusion events automatically.

\subsubsection{Decision Tree (DT)}

Quinlan [43] defined Decision Trees as "powerful and common tools for classification and prediction. A decision tree is a tree that has three main components: nodes, arcs and leaves. Each node is labeled with a feature attribute, which is most informative among the attributes not yet considered in the path from the root. Each arc out of a node is labeled with a feature value for the node's feature, and each leaf is labeled with a category or class. A decision tree can then be used to classify a data point by starting at the root of the tree and moving through it until a leaf node is reached. The leaf node provides the classification of the data point. ID3 and C4.5 developed by Quinlan are the most common implementations of the Decision Tree."

Peddabachigari et al. [41], proposed decision trees (DT) and support vector machines (SVM) as intrusion-detection model. 
They also designed a hybrid DT-SVM model and an ensemble approach with decision tree where SVM and DT-SVM models proposed as base classifiers. Joong et al. [23] generated the decision trees for DoS attacks, R2L attack, U2R attack, and Scan attack. The ID3 algorithm is used as the learning algorithm to generate the decision tree automatically.

\subsubsection{Support Vector Machine (SVM)}

Support vector machines (SVM) are proposed by Vapnik [56]. SVM first maps the input vector into a higher-dimensional feature space and then obtains the optimal separating hyperplane in the high dimensional feature space. Moreover, a decision boundary, i.e. the separating hyper-plane, is determined by support vectors rather than the whole training samples and thus is extremely robust to outliers. In particular, an SVM classifier is designed for binary classification. That is, to separate a set of training vectors, which belong to two different class's notes that the support vectors are the training samples close to a decision boundary. The SVM also provides a user-specified parameter called a penalty factor. It allows users to make a tradeoff between the number of misclassification samples and the width of a decision boundary.

Mukkamala et al. [37] designed model to network anomaly detection problems by "applied kernel classifiers and classifier design methods to network anomaly detection problems. They evaluated the impact of kernel type and parameter values on the accuracy with which a support vector machine (SVM) performs intrusion classification. Jun et al. [25] proposed PSO-SVM model is applied to an intrusion detection problem, the standard PSO is used to determine free parameters of support vector machine and the binary PSO is to obtain the optimum feature subset at the building intrusion detection system. Paulo et al. [40] proposed an intrusion detection system model based on the behavior of network traffic through the analysis and classification of messages. Two artificial intelligence techniques named Kohonen neural network (KNN) and support vector machine (SVM) are applied to detect anomalies.

\subsection{Unsupervised Anomaly Detection Techniques}

These techniques do not need training data. As alternative, they based on two basic assumptions. First, they presume that most of the network connections are normal traffic and only a very small traffic percentage is abnormal. Second, they anticipate that malicious traffic is statistically various from normal traffic. According to these two assumptions, data groups of similar instances which appear frequently are assumed to be normal traffic, while infrequently instances which considerably various from the majority of the instances are regarded to be malicious [7]. The most common unsupervised algorithms are, K-Means, Self-organizing maps (SOM), C-means, Expectation-Maximization Meta algorithm (EM), Adaptive resonance theory (ART), Unsupervised Niche Clustering (UNC) and One-Class Support Vector Machine.

\subsubsection{Clustering Techniques}

Rawat [45] and many more found that Clustering techniques work by grouping the observed data into clusters, according to a given similarity or distance measure. There exist at least two approaches to clustering based anomaly detection. In the first approach, the anomaly detection model is trained using unlabeled data that consist of both normal as well as attack traffic. In the second approach, the model is trained using only normal data and a profile of normal activity is created. The idea behind the first approach is that anomalous or attack data forms a small percentage of the total data. If this assumption holds, anomalies and attacks can be detected based on cluster sizes large clusters correspond to normal data, and the rest of the data points, which are outliers, correspond to attacks.

\subsubsection{Unsupervised Neural Network}

The two typical unsupervised neural networks are selforganizing maps and adaptive resonance theory. They used similarity to group objects. They are adequate for intrusion detection tasks where normal behavior is densely concentrated around one or two centers, while anomaly behavior and intrusions spread in space outside of normal clusters.

The Self-organizing map (SOM) is trained by an unsupervised competitive learning algorithm [26]. The aim of the SOM is to reduce the dimension of data visualization. That is, SOM outputs are clustered in a low dimensional (usually 2D or 3D) grid. It usually consists of an input layer and the Kohonen layer, which is designed as the two-dimensional arrangement of neurons that maps $\mathrm{n}$ dimensional input to two dimensions. Kohonen's SOM associates each of the input vectors to a representative output. The network finds the node nearest to each training case and moves the winning node, which is the closest neuron (i.e. the neuron with minimum distance) in the training course. That is, SOM maps similar input vectors onto the same or similar output units on such a two-dimensional map, which leads to self-organize the output units into an ordered map and the output units of similar weights are also placed nearby after training.

SOMs are the most popular neural networks to be trained for anomaly detection tasks. For example Kayacik et al. [28], they have created three layers of employment: First, individual SOM is associated with each basic TCP feature. Second layer integrates the views provided by the first-level SOM into a single view of the problem. The final layer is built for those neurons, which win for both attack and normal behaviors. Oh and Chae [39] proposed an approach a real-time intrusiondetection system based on SOM that groups similar data and visualizes their clusters. The system labels the map produced by SOM using correlations between features. Jun et al. [24] introduced a novel methodology to analysis the feature attributes of network traffic flow with some new techniques, including a novel quantization model of TCP states. Integrating with data preprocessing, the authors construct an anomaly detection algorithm with SOFM and applied the detection frame to DARPA Intrusion Detection Evaluation Data.

Adaptive Resonance Theory (ART). The adaptive resonance theory embraces a series of neural network models that perform unsupervised or supervised learning, pattern recognition, and prediction. Unsupervised learning models Include ART-1, ART- 2, ART-3, and Fuzzy ART. Various supervised networks are named with the suffix "MAP", such as ARTMAP, Fuzzy ARTMAP, and Gaussian ARTMAP.

Amini et al. [1] Compared the performance of ART-1 (accepting binary inputs) and ART-2 (accepting continuous inputs) on KDD99 data. Liao et al. [29] deployed Fuzzy ART in an adaptive learning framework which is suitable for dynamic changing environments. Normal behavior changes are efficiently accommodated while anomalous activities can still be identified.

\subsubsection{K-Means}

$\mathrm{K}$-means algorithm is a traditional clustering algorithm. It divides the data into $\mathrm{k}$ clusters, and guarantee that the data within the same cluster are similar, while the data in a various 
clusters have low similarities. K-means algorithm is first selected $\mathrm{K}$ data at random as the initial cluster center, for the rest data add it to the cluster with the highest similarity according to its distance to the cluster center, and then recalculate the cluster center of each cluster. Repeat this process until each cluster center doesn't change. Thus data are divided into $\mathrm{K}$ clusters. Unfortunately, $\mathrm{K}$-means clustering is sensitive to the outliers and a set of objects closer to a centroid may be empty, in which case centroids cannot be updated [16].

[30] proposed K-means algorithms for anomaly detection. Firstly, a method to reduce the noise and isolated points in the data set was advanced. By dividing and merging clusters and using the density radius of a super sphere, an algorithm to calculate the number of the cluster centroid was given. By more accurate method of finding $\mathrm{k}$ clustering center, an anomaly detection model was presented to get better detection effect. Cuixiao et al. [7] proposed a mixed intrusion detection system (IDS) model. Data are examined by the misuse detection module and then the detection of abnormal data is performed by anomaly detection module. In this model, unsupervised clustering method is used to build the anomaly detection module. The algorithm used is an improved algorithm of K-means clustering algorithm and it is demonstrate to have a high detection rate in the anomaly detection module.

\subsubsection{Fuzzy C-Means (FCM)}

Fuzzy C-means is a clustering method, which grants one piece of data to belong to two or more clusters. It was developed by Dunn [9] and improved later by Bezdek [3], it is used in applications for which hard classification of data is not meaningful or difficult to achieve (e.g, pattern recognition). C-means algorithm is similar to K-Means except that membership of each point is defined based on a fuzzy function and all the points contribute to the relocation of a cluster centroid based on their fuzzy membership to that cluster.

Shingo et al. [52] proposed a new approach called FC-ANN, based on ANN and fuzzy clustering to solve the problem and help IDS achieving higher detection rate, less false positive rate and stronger stability. Yu and Jian [58] proposed an approach integrating several soft computing techniques to build a hierarchical neuro-fuzzy inference intrusion detection system. In this approach, principal component analysis neural network is used to reduce feature space dimensions. The preprocessed data were clustered by applying an enhanced fuzzy C-means clustering algorithm to extract and manage fuzzy rules. Another approach that uses a fuzzy approach for unsupervised clustering is presented by Shah et al. [50]. They employed the Fuzzy C-Medoids (FCMdd) in order to index cluster streams of system call, low level Kernel data and network data.

\subsubsection{Unsupervised Niche Clustering (UNC)}

(UNC) is a robust clustering algorithm, which uses an evolutionary algorithm with a niching strategy (Nasraoui et al. [38]. The evolutionary algorithm helps to find clusters using a robust density fitness function, while the niching technique allows it to create and maintain the niches (candidate clusters). Since UNC is based on genetic optimization, it is much less susceptible to suboptimal solutions than traditional techniques. The algorithm main advantage is the ability to handle noise and to determine clusters number automatically.
Elizabeth et al. [10] combined the UNC with fuzzy set theory for anomaly detection and applied it to network intrusion detection. They associated to each cluster generated by the UNC a member function that follows a Gaussian shape using evolved cluster center and radius. Such cluster membership functions will define the normalcy level of a data sample.

\subsubsection{Expectation-Maximization Meta Algorithm $(E M)$}

EM is another soft clustering method based on ExpectationMaximization Meta algorithm Dempster et al. [8]. Expectation-Maximization is an algorithm for finding maximum probability estimates of parameters in probabilistic models. EM clustering algorithm alternates between performing expectation (E) step, by computing an estimation of likelihood using current model parameters (as if they are known), and a maximization (M) step, by computing the maximum probability estimates of model parameters. The model parameters new estimations contribute to an expectation step of next iteration.

Hajji [15] used Gaussian mixture models to characterize utilization measurements. Model parameters are estimated using Expectation-Maximization (EM) algorithm and anomalies are detected corresponding to network failure events. Animesh and Jung [2] proposed an anomaly detection scheme, called SCAN to address the threats posed by network-based denial of service attacks in high speed networks. The noteworthy features of SCAN include: (a) it rationally samples the incoming network traffic to reduce the amount of audit data being sampled while retaining the intrinsic characteristics of the network traffic itself; (b) it computes the missing elements of the sampled audit data by using an enhanced Expectation-Maximization (EM) algorithm-based clustering algorithm; and (c) it enhances the convergence speed of the clustering process by employing Bloom filters and data summaries.

\subsubsection{One -Class Support Vector Machine (OCSVM)}

The one-class support vector machine is a very specified sample of a support vector machine which is geared for anomaly detection. The one-class SVM varies from the SVM generic version in that the resulting problem of quadratic optimization includes an allowance for a specific small predefined outliers percentage, making it proper for anomaly detection. These outliers lie between the origin and the optimal separating hyper plane. All the remaining data fall on the opposite side of the optimal separating hyper plane, belonging to a single nominal class, hence the terminology "one-class" SVM. The SVM outputs a score that represents the distance from the data point being tested to the optimal hyper plane. Positive values for the one-class SVM output represent normal behavior (with higher values representing greater normality) and negative values represent abnormal behavior (with lower values representing greater abnormality) [42].

Eskin et al. [11] and Honig et al. [19] used an SVM in addition to their clustering methods for unsupervised learning. The SVM algorithm had to be modified a little to work in unsupervised learning domain. Once it was, it performs better than both of their clustering methods. 
Shon and Moon [53] suggested a new SVM approach, named Enhanced SVM, which merges (soft-margin SVM method and one-class SVM) in order to provide unsupervised learning and low false alarm capability, similar to that of a supervised SVM approach. Rui et al. [46] proposed a method for network anomaly detection based on one class support vector machine (OCSVM). The method contains two main steps: first is the detector training, the training data set is used to generate the OCSVM detector, which is capable to learn the data nominal profile, and the second step is to detect the anomalies in the performance data with the trained detector.

\subsection{Anomaly Detection Algorithms Comparison}

Various unsupervised anomaly detection algorithms have been applied to intrusion detection to enhance IDSs performance in all levels such as in clustering, features selection and classifications. Based on the previous description of the different unsupervised anomaly detection algorithms, Table 1 shows a comparison among the most common algorithms. The comparison summarizes the pros and cons of each one.

the weaknesses of knowledge base detection techniques. Anomaly detection comprises supervised techniques and unsupervised techniques. Many algorithms were used to achieve good results for these techniques. This paper proposes an overview of machine learning techniques for anomaly detection. The experiments demonstrated that the supervised learning methods significantly outperform the unsupervised ones if the test data contains no unknown attacks. Among the supervised methods, the best performance is achieved by the non-linear methods, such as SVM, multi-layer perceptron and the rule-based methods. Techniques for unsupervised such as K-Means, SOM, and one class SVM achieved better performance over the other techniques although they differ in their capabilities of detecting all attacks classes efficiently.

Table 1: Pros and Cons of Anomaly Detection Techniques

\begin{tabular}{|c|l|l|}
\hline Technique & \multicolumn{1}{|c|}{ Pros } & \multicolumn{1}{c|}{ Cons } \\
\hline $\begin{array}{c}\text { K -Nearest } \\
\text { Neighbor }\end{array}$ & $\begin{array}{l}\text { Very easy to } \\
\text { understand when } \\
\text { there are few } \\
\text { predictor } \\
\text { variables. } \\
\begin{array}{l}\text { Useful for } \\
\text { building models } \\
\text { that involve non- } \\
\text { standard data } \\
\text { types, such as text. }\end{array}\end{array}$ & $\begin{array}{l}\text { Have large storage } \\
\text { requirements. } \\
\text { Sensitive to the choice of } \\
\text { the similarity function that is } \\
\text { used to compare instances. } \\
\text { Lack a principled way to } \\
\text { choose k, except through } \\
\text { cross-validation or similar. } \\
\text { Computationally-expensive } \\
\text { technique. }\end{array}$ \\
\hline $\begin{array}{c}\text { A neural network } \\
\text { Neural } \\
\text { can perform tasks } \\
\text { that a linear } \\
\text { program cannot. } \\
\text { When an element } \\
\text { of the neural } \\
\text { network fails, it } \\
\text { can continue } \\
\text { without any }\end{array}$ & $\begin{array}{l}\text { The neural network needs } \\
\text { training to operate. } \\
\text { The architecture of a neural } \\
\text { network is different from the } \\
\text { architecture of } \\
\text { microprocessors therefore } \\
\text { needs to be emulated. } \\
\text { Requires high processing } \\
\text { time for large neural }\end{array}$ \\
\hline
\end{tabular}

\begin{tabular}{|c|c|c|}
\hline & $\begin{array}{l}\text { problem with their } \\
\text { parallel nature. } \\
\text { A neural network } \\
\text { learns and does } \\
\text { not need to be } \\
\text { reprogrammed. } \\
\text { It can be } \\
\text { implemented in } \\
\text { any application. } \\
\end{array}$ & networks. \\
\hline $\begin{array}{c}\text { Decision } \\
\text { Tree }\end{array}$ & $\begin{array}{l}\text { Simple to } \\
\text { understand and } \\
\text { interpret. } \\
\text { Requires little } \\
\text { data preparation. } \\
\text { Able to handle } \\
\text { both numerical } \\
\text { and categorical } \\
\text { data. } \\
\text { Uses a white box } \\
\text { model. } \\
\text { Possible to } \\
\text { validate a model } \\
\text { using statistical } \\
\text { tests. } \\
\text { Robust. } \\
\text { Perform well with } \\
\text { large data in a } \\
\text { short time. }\end{array}$ & $\begin{array}{l}\text { The problem of learning an } \\
\text { optimal decision tree is } \\
\text { known to be NP-complete } \\
\text { under several aspects of } \\
\text { optimality and even for } \\
\text { simple concepts. } \\
\text { Decision-tree learners create } \\
\text { over-complex trees that do } \\
\text { not generalize the data well. } \\
\text { There are concepts that are } \\
\text { hard to learn because } \\
\text { decision trees do not express } \\
\text { them easily. }\end{array}$ \\
\hline $\begin{array}{l}\text { Support } \\
\text { Vector } \\
\text { Machine }\end{array}$ & $\begin{array}{l}\text { Find the optimal } \\
\text { separation hyper } \\
\text { plane. } \\
\text { Can deal with } \\
\text { very high } \\
\text { dimensional data. } \\
\text { Some kernels } \\
\text { have infinite } \\
\text { Vapnik- } \\
\text { Chervonenkis } \\
\text { dimension, which } \\
\text { means that they } \\
\text { can learn very } \\
\text { elaborate } \\
\text { concepts. } \\
\text { Usually work } \\
\text { very well. }\end{array}$ & $\begin{array}{l}\text { Require both positive and } \\
\text { negative examples. } \\
\text { Need to select a good kernel } \\
\text { function. } \\
\text { Require lots of memory and } \\
\text { CPU time. } \\
\text { There are some numerical } \\
\text { stability problems in solving } \\
\text { the constraint } \\
\text { QP. }\end{array}$ \\
\hline $\begin{array}{c}\text { Self- } \\
\text { organizing } \\
\text { map }\end{array}$ & $\begin{array}{l}\text { Simple and easy- } \\
\text { to-understand } \\
\text { algorithm that } \\
\text { works. } \\
\text { A topological } \\
\text { clustering } \\
\text { unsupervised } \\
\text { algorithm that } \\
\text { works with } \\
\text { nonlinear data set. } \\
\text { The excellent } \\
\text { capability to } \\
\text { visualize high- } \\
\text { dimensional data } \\
\text { onto } 1 \text { or } 2 \\
\text { dimensional space }\end{array}$ & Time consuming algorithm \\
\hline
\end{tabular}




\begin{tabular}{|c|l|l|}
\hline & $\begin{array}{l}\text { makes it unique } \\
\text { especially for } \\
\text { dimensionality } \\
\text { reduction. }\end{array}$ & \\
\hline K-means & Low complexity. & $\begin{array}{l}\text { Necessity of specifying k. } \\
\text { Sensitive to noise and outlier } \\
\text { data points. } \\
\text { Clusters are sensitive to } \\
\text { initial assignment of } \\
\text { centroids. }\end{array}$ \\
\hline Fuzzy C- \\
means & $\begin{array}{l}\text { Allows a data } \\
\text { point to be in } \\
\text { multiple clusters. } \\
\text { A more natural } \\
\text { representation of } \\
\text { the behavior of } \\
\text { genes. }\end{array}$ & $\begin{array}{l}\text { Need to define c, the clusters } \\
\text { number. } \\
\text { Need to determine } \\
\text { membership cutoff value. } \\
\text { Clusters are sensitive to } \\
\text { initial assignment of } \\
\text { centroids. }\end{array}$ \\
\hline $\begin{array}{c}\text { Expectation- } \\
\text { Maximization } \\
\text { Meta }\end{array}$ & $\begin{array}{l}\text { Can easily change } \\
\text { the model to adapt } \\
\text { to a different } \\
\text { distribution of data } \\
\text { sets. } \\
\text { Parameters } \\
\text { number does not } \\
\text { increase with the } \\
\text { training data } \\
\text { increasing. }\end{array}$ & $\begin{array}{l}\text { Slow convergence in some } \\
\text { cases }\end{array}$ \\
\hline
\end{tabular}

\section{CONCLOUSION}

Machine learning techniques have received considerable attention among the intrusion detection researchers to address the weaknesses of knowledge base detection techniques. Anomaly detection comprises supervised techniques and unsupervised techniques. Many algorithms were used to achieve good results for these techniques. This paper proposes an overview of machine learning techniques for anomaly detection. The experiments demonstrated that the supervised learning methods significantly outperform the unsupervised ones if the test data contains no unknown attacks. Among the supervised methods, the best performance is achieved by the non-linear methods, such as SVM, multi-layer perceptron and the rule-based methods. Techniques for unsupervised such as K-Means, SOM, and one class SVM achieved better performance over the other techniques although they differ in their capabilities of detecting all attacks classes efficiently.

\section{REFERENCES}

[1] Amini and Jalili. 2004. Network-based intrusion detection using unsupervised adaptive resonance theory. in Proceedings of the 4th Conference on Engineering of Intelligent Systems (EIS'04).

[2] Animesh, P. and Jung,M. 2007. "Network Anomaly Detection with Incomplete Audit Data". Elsevier Science, 12 February, 2007, pp. 5-35.

[3] Bezdek, J. 1981." Pattern recognition with fuzzy objective function algorithms". Kluwer Academic Publishers, Norwell, MA, USA (1981).

[4] Bishop, C.1995. Neural networks for pattern recognition England, Oxford University.
[5] Bouzida, F., Cuppens,B. and Gombault,s.2004.Efficient intrusion detection using principal component analysis. in Proceedings of the 3ème Conférence sur la Sécurité et Architectures Réseaux (SAR).

[6] Chan, F. , Yeung,S. and Tsang,S.2005. Comparison of different fusion approaches for network intrusion detection using an ensemble of RBFNN. in: Proceedings of 2005 International Conference on Machine Learning and Cybernetics.

[7] Guobing,Z.,Cuixia,Z.and Shanshan,s.2009. A Mixed Unsupervised Clustering-based Intrusion Detection Model. Third International Conference on Genetic and Evolutionary Computing.

[8] Dempster,A., Laird, N.and Rubin, D. 1977." Maximum likelihood from incomplete Data via the EM algorithm". J. Royal Stat, Soc, Vol. 39, 1977, pp. 1-38.

[9] Dunn, J. 1973." A fuzzy relative of the ISO data process and its use in detecting compact well-separated clusters". Journal of Cyber natics, Vol.3(3), pp. 32-57.

[10] Lizabeth, L., Olfa, N. and Jonatan,G.2007. Anomaly detection based on unsupervised niche clustering with application to network intrusion detection. Proceedings of the IEEE Conference on Evolutionary Computation.

[11] Eskin,E.,Arnold,A .,Preraua,M., Portnoy.L and Stolfo,S.” A geometric framework for unsupervised anomaly detection: Detecting intrusions in unlabeled data”. In D. Barber and S. Jajodia (Eds.). Data Mining for Security Applications. Boston: Kluwer Academic Publishers.

[12] Estevez,J.,Garcya,P. and Dyaz, J. 2004.”Anomaly detection methods in wired networks: a survey and taxonomy". Computer Networks. Vol .27, No.16, 2004, pp. 1569-84.

[13] Garcia,T. Diaz,V. Macia,F. and Vazquezb. 2009. "Anomaly-based network intrusion detection". Computers and security, Vol. 2 8, 2009 , pp. $18-28$.

[14] Gilles, C., Melanie, H. and Christian,P. 2004.” One-class support vector machines with a conformal kernel". A case study in handling class imbalance .In Structural Syntactic and Statistical Pattern Recognition, 2004, pp.850-858.

[15] Hajji ,H." Statistical Analysis of Network Traffic for Adaptive Faults Detection”. 2005. IEEE Trans. Neural Networks, Vol.16, NO5, 2005, PP. 1053-1063.

[16] Han, J. and Kamber, M. 2001.” Data mining: Concept and Techniques. (1th Ed), Morgan Kaufman publishers,

[17] Heckerman 1995." A tutorial on Learning with Bayesian Networks". Technical report. Microsoft research, MSRTR, Vol 6

[18] Hofmann,A., Schmitz,C. and Sick, B.2003. Rule extraction from neural networks for intrusion detection in 
computer networks.in IEEE International Conference on Systems, Man and Cybernetics.

[19] Honig, A. 2002" Adaptive model generation: An architecture for the deployment of data mining based intrusion detection systems". In D. Barbar and S. Jajodia, (Eds.), Data Mining for Security Applications. Boston: Kluwer Academic Publishers May 2002.

[20] Jain, A., Murty, M. and Flynn, P. 1999.” Data clustering: A review". ACM Computing Surveys, Vol. 31, NO3, pp. 264-323.

[21] Jiang,J.,Zhang,C. and Kame,M.2003. RBF-based realTime hierarchical intrusion detection systems. In Proceedings of the International Joint Conference on Neural Networks (IJCNN'03).

[22] Johansen, K. and Lee. " CS424 network security: Bayesian Network Intrusion Detection (BINDS)": http://citeseerx.ist.psu.edu/viewdoc/summary?doi=10.1.1 .83 .8479

[23] Joong, L., Jong,H., Seon,G. and Tai,M . 2008." Effective Value of Decision Tree with KDD99 Intrusion Detection Datasets for Intrusion Detection System". ICACT, pp. 17-20.

[24] Jun, Z., Ming, H., Hong, Z .2004. A new Method of Data Preprocessing and Anomaly Detection. Pro. of Third Inter. Conf on Machine Learning and cybernetics.

[25] Jun, W., Xu,H.,Rong, R. and Tai-hang ,L.2009. A Real Time Intrusion Detection System Based on PSO-SVM. Proceedings of the International Workshop on Information Security and Application (IWISA).

[26] Kohonen, 1995.” Self-Organizing Map”. Springer, Berlin,

[27] Kumar, G. Kumar, K. and Sachdeva, M.2010. The use of artificial intelligence based techniques for intrusion detection: a review.

[28] Kayacik, G., Zincir, H. and Heywood, M.2003. On the Capability of an SOM Based Intrusion Detection System. Proc IEEE, IJCNN.

[29] Liao,Y. , Vemuri,R. and Pasos,A. 2007." Adaptive anomaly detection with evolving connectionist Systems". Journal of Network and Computer Applications, Vol.30, NO1, PP. 60-80.

[30] [30] LI,H 2010.Research and Implementation of an Anomaly Detection Model Based on Clustering Analysis. International Symposium on Intelligent Information Processing and Trusted Computing.

[31] Liu,Z., Florez, C. and Bridges, S.2002. A comparison of input representations in neural networks: a case study in intrusion detection. In Proceedings of the International Joint Conference on Neural Networks (IJCNN'02).
[32] Manocha, S. and Girolami, M. 2007." An empirical analysis of the probabilistic K-nearest Neighbor Classifier". Pattern Recognition Letters, Vol. 28, pp. $1818-1824$

[33] Ming, Y. 2011. " Real Time Anomaly Detection Systems for Denial of Service Attacks by Weighted k-NearestNeighbor Classifiers". Expert Systems with Applications, Vol.38, 2011, pp. 3492-3498.

[34] Mohammed,.S., Marwa, S., Mohammed, Imane, S.2007.Artificial Neural Networks Architecture for IntrusionDetection Systems and Classification of Attacks, CairoUniversity, Egypt.

[35] Moore, D.2005. Internet Traffic Classification Using Bayesian Analysis Techniques. in Proceedings of ACM SIGMETRICS.

[36] Moradi and Zulkernine.2004. A Neural Network Based System for Intrusion Detection and Classification of Attacks.IEEE International Conference on Advances in Intelligent Systems-Theory and Applications, Luxembourg: Kirchberg.

[37] Mukkamala,S.,Sung, A.and Ribeiro, B.2005. Model Selection for Kernel Based Intrusion Detection Systems. Proceedings of International Conference on Adaptive and Natural Computing Algorithm.

[38] Nasraoui, O., Leon, E. \& Krishnapuram, R. 2005. Unsupervised Niche Clustering: Discovering an Unknown Number of Clusters in Noisy Data Sets. In: GHOSH, A. \& JAIN, L. (eds.) Evolutionary Computation in Data Mining. Springer Berlin Heidelberg.

[39] Oh and Chae.2008. Real Time Intrusion Detection System Based on Self-Organized Maps and Feature Correlations. The Proceedings of the Third International Conference on Convergence and Hybrid Information.

[40] Paulo, M., Vinicius , M. and Joni.2010. Octopus-IIDS: An Anomaly Based Intelligent Intrusion Detection System.Proceedings of Computers and Communications (ISCC).

[41] Peddabachigari, S., Abraham, A., Grosan, C. and Thomas, J. 2007." Modeling Intrusion Detection System using Hybrid Intelligent Systems". J. Netw. Comput. Appl, Vol. 30, NO1, PP. 114-132.

[42] Gilles,C.,Melanie, H. and Christian, P.2004.One-Class Support vector Machines with a Conformal kernel A case study in handling class Imbalance. In: Structural yntactic and Statistical Pattern Recognition.

[43] Quinlan, J.1993." C4.5: programs for machine learning". Log Altos, CA, Morgan Kaufmann.

[44] Rapaka,A., Novokhodko,A. and Wunsch,D.2003 Intrusion detection using radial basis function network on sequence of system calls. In Proceedings of theInternational Joint Conference on Neural Networks (IJCNN'03).

[45] Rawat,S.2005. Efficient Data Mining Algorithms for Intrusion Detection. in Proceedings of the 4th 
Conference on Engineering of Intelligent Systems (EIS'04).

[46] Rui, Z., Shaoyan, Z., Yang, L. and Jianmin ,J.2008. Network Anomaly Detection Using One Class Support Vector Machine. Proceedings of the International Multi Conference of Engineers and Computer Scientists.

[47] Rumelhart, D. Hinton, G. and Williams, R. 1986. " Learning internal representations by error propagation" . In: Rumelhart, D., McClelland J L et al. (Eds.) Parallel Distributed Processing: Explorations in theMicrostructure of Cognition. MIT Press, Cambridge, MA,Vol. 1, pp. 318-362.

[48] Sahar, S., Hashem, M. and Taymoor, M. 2010." Intrusion Detection using Multi-Stage". Neural Network. International Journal of Computer Science and Information Security, Vol. 8, NO 4, PP. 14-20.

[49] Santanu, D., Ashok, S. and Aditi, C.2007. Classification of Damage Signatures in Composite Plates using OneClass SVM's. In Proceedings of the IEEE Aerospace Conference, Big Sky. MO.

[50] Shah,H., Undercoffer,J. and Joshi, A. 2003. Fuzzy Clustering for Intrusion Detection. the 12th IEEE International Conference on Fuzzy Systems.

[51] Shailendra and Sanjay. 2009." An ensemble approach for feature selection of Cyber Attack Dataset", International Journal of Computer Science and Information Security P12-(IJCSIS), Vol.6, NO 2.

[52] Shingo, M., Ci, C. Nannan, L. Kaoru, S. and Kotaro, H.” An Intrusion Detection Model Based on Fuzzy Class Association Rule Mining Using Genetic Network
Programming”. IEEE Transactions on Systems, Part C. Vol.41, pp. 130-139.

[53] Shon and Moon. 2007." A hybrid Machine Learning Approach to Network Anomaly Detection". Inf. SCI, Vol.177, NO 18, PP. 3799-3821.

[54] Srinivas, M. 2002. Intrusion Detection using Neural Networks and Support vector Machine. Proceedings of the IEEE International HI.

[55] Theodoridis, S. and Koutroumbas. 2006. "Pattern recognition (3rd Ed.)". USA: Academic Press.

[56] Vapnik, V." Statistical learning theory”. Wiley, New York, 1998

[57] Yao,Y. , Wei, Y. GAO, F. and Yu,G.2006. Anomaly Intrusion Detection Approach Using Hybrid MLP/CNN Neural Network. Proceedings of the Sixth International Conference on Intelligent Systems Design and Applications.

[58] Yu, Z. and Jian, F. 2009 Intrusion Detection Model Based on Hierarchical Fuzzy Inference System. Second International Conference on Information and Computing Science Icic.

[59] Peng, N. and Sushil, J. 2003. "Intrusion Detection Techniques". nIn H. Bidgoli (Ed.), the Internet Encyclopedia. John Wiley \& Sons.

[60] Ghorbani, Wei and Tavallaee. 2010." Theoretical Foundation of Detection Network Intrusion Detection and Prevention". Concepts and Techniques Advances in Information Security. Springer Science,Vol.47, pp.47114. 